\title{
PREDICCIÓN DEL TIEMPO DE CONGELACIÓN DE ALIMENTOS CON EL MÉTODO DE MANNAPERUMA
}

Ricardo Carranza de la Torre ${ }^{\prime}$

\author{
RE S U M E N
}

Se utilizó la formulación numérica explícita de diferencias finitas de Mannaperuma en la predicción de los tiempos de congelación y descongelación de caracol, abalón, bacalao, carne de res y otros alimentos; la contrastación de estas predicciones con experimentos reportados en la bibliografía indicó que el programa tiende a sobrestimar aparentemente los tiempos de congelación para criterios de terminación de temperatura en el centro y subestimar los de descongelación para criterios de terminación basados en temperatura de equilibrio. Se obtuvieron también tablas de propiedades térmicas simuladas para cada especie.

\section{A B S T R A C T}

The explicit numeric formulation of finite differences of Mannaperuma was used in the prediction of the times of freezing and thawing of snail, abalón, cod, beef and other foods; the comparison of these predictions with experiments reported in the bibliography indicated that the program apparenthy tends to overestimate the times of freezing for approaches of termination of temperature in the center, and to underestimate those of thawing for termination approaches based on balance temperature. Also, charts of simulated thermal properties were obtained for each species.

\section{INTRODUCCIÓN}

El tiempo de congelación es un factor importante en el diseño de planta y el planeamiento de la producción, pues su conocimiento implica ahorros y asegura la calidad de los alimentos congelados. Hay métodos de predicción de varios tipos que van desde los más simples de fórmula teórica como el clásico de Plank, los de fórmula semiempírica como el Nagaoka hasta los más complicados de soluciones matemáticas exactas de la ecuación de conducción de calor con cambio de fase. También las soluciones numéricas de esta última ecuación han sido materia de estudio en los últimos 30 años a nivel mundial; en esta área se han desarrollado métodos de aceptable exactitud como los gráficos, los de fórmula explícita

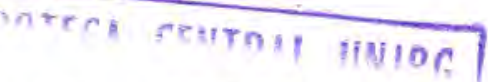

en diferencias finitas, los de fórmula implícita del tipo Crank Nicholson y de tres subniveles de tiempo de Bonacina y Comini; mención aparte requieren las técnicas de elementos finitos que eliminan las limitaciones de las soluciones numéricas anteriores. Entre las formulaciones explícitas en diferencias finitas está la de Mannaperuma - Heldman que utiliza la entalpía como dato base para la predicción interpolando valorescorrespondientes de ella ala temperatura que los nodos considerados en el cuerpo tengan en todo momento; esta formulación entálpica salva el problema de la fuerte discontinuidad que se presenta cuando se usa la formulación del calor específico aparente.

I Magister en Ingeniería de Procesos de Alimentos. 
Todos los métodos usados para la predicción del tiempo de congelación requieren ser validados contra datos experimentales publicados sobre sustancias homogéneas como la tilosa o contra experimentación directa con ellas en congelación y descongelación, asimismo, su contrastación con datos experimentales obtenidos con alimentos es de gran importancia toda vez que ellos distan de ser sustancias simples y homogéneas. El método presentado por Mannaperuma - Heldman no ha sido comparado con experiencias de congelación/descongelación de alimentos, pero permite, vía la elaboración de tablas de propiedades físicas, simular esos procesos añadiendo al trabajo de diseño de plantas y producción, la ventaja de la reducción de la inversión en el de otro modo trabajo exclusivo de experimentación en planta piloto. En Tacna existen varias congeladoras de mariscos en las que la tecnología permite congelar en tiempos demasiado largos que afectan la calidad de esos alimentos y una propuesta técnica que, aunque implique gastos de renovación de equipos, signifique a la larga una mejora de importancia en sus tareas de procesamiento, bien puede valer la pena en términos de la calidad y prestigio de los productos que actualmente se exportan al Oriente y USA.

El objetivo general de este trabajo es aplicar el método de Mannaperuma que es suficientemente exacto y de fácil uso para la predicción del tiempo de congelación y descongelación de alimentos a productos alimenticios congelados peruanos

Y los específicos son:

- Elaborar tablas de propiedades físicas de abalones, caracoles y productos cárnicos.

- Elaborartablas de entalpía de abalones, caracoles y productos cárnicos.

- Calcular el tiempo de congelación de productos como abalones, caracoles y productos cárnicos.

- Calcular el tiempo de descongelación de esos mismos productos.

\section{MATERIALES Y MÉTODOS}

El estudio consistió primero en conocer los alcances del método de Mannaperuma en términos de sus significados físico y matemático.

\section{DESCRIPCIÓN Y FORMULACIÓN MATEMÁTICA}

La congelación y descongelación en un material alimenticio puede tratarse como conducción de calor con cambio de fase. El cambio de fase real tiene lugar en un amplio rango de temperatura y las propiedades alimenticias cambian considerablemente en ese rango. Este cambio gradual de fase puede incorporarse en el proceso de conducción de calor de varias maneras. Dos métodos posibles son la formulación del calor específico aparente y la formulación entálpica.

\section{FORMULACIÓN DEL CALOR ESPECÍFICO APARENTE}

Incorpora la entalpía del cambio de fase dentro de una adecuadamente definida "Capacidad calórica específica aparente".

Permite también el uso de la misma ecuación para ambas fases por incorporación implicita de la condición de frontera móvil en la ecuación gobernante:

$$
C(T) \cdot \frac{\partial T}{\partial t}=\nabla \cdot[K(T) \nabla T]
$$

Las propiedades térmicas se vuelven dependientes de la temperatura al permitirles extenderse en el cambio de fase; estas dependencias son no lineales y muestran discontinuidades en el punto inicial de congelación que evitan solución analitica de la ecuación gobernante.

La solución numérica explícita en diferencias finitas es sencilla pero tiene la limitación de los criterios de estabilidad que restringen el incremento de tiempo y por las oscilaciones causadas por el pico agudo de la función del calor específico aparente a la temperatura del cambio de fase inicial.

Las soluciones numéricas totalmente implícitas y las del tipo Crank-Nicholson dan como resultado sistemas de ecuaciones no lineales que requieren métodos de solución iterativos consumidores de tiempo. Lees (1966) propuso un esquema lineal de diferencias finitas de tres niveles de tiempo para vencer estas dificultades: 


$$
\begin{gathered}
C\left[T_{i}^{j}\right] \frac{\left[T_{i}^{j+1}-T_{i}^{j-1}\right]}{2 \Delta t}=\frac{1}{\Delta x}\left[K\left(T_{i+1 / 2}^{j}\right)\right] \\
{\left[\frac{\left.\bar{T}_{i+1}-\bar{T}_{i}\right]}{\Delta x}-\left[K\left(T_{i-1 / 2}^{j}\right)\right]-\frac{\left[\bar{T}_{i}-\bar{T}_{i-1}\right]}{\Delta x}\right]}
\end{gathered}
$$

donde:

$$
\begin{aligned}
\bar{T}_{i} & =\frac{1}{3} \cdot\left[T_{i}^{j+1}+T_{i}^{j}+T_{i}^{j-1}\right] \\
\mathrm{i} & =\text { punto nodal } \\
\mathrm{j} & =\text { incremento de tiempo }
\end{aligned}
$$

Este esquema origina un sistema lineal de ecuaciones con las temperaturas incógnitas en el nivel de tiempo $(j+1)$ ésimo y pueden resolverse usando procedimientos estándares de eliminación. Cleland y Earle (1977) aplicaron este esquema bajo condiciones de frontera convectiva derivando la ecuación para el nodo de frontera extendiendo una placa de tilosa medio elemento y escribiendo el balance de calor para el elemento de frontera extendida. Observaron oscilaciones cuando la diferencia de temperatura eran grandes y remediaron la situación modificando la actualización de la temperatura a partir de:

$$
T_{i}^{j} \rightarrow T_{i}^{j-1} a \frac{1}{3}\left[T_{i}^{j+1}+T_{i}^{j}+T_{i}^{j-1}\right]-T_{i}^{j-1}
$$

Manifestaron entonces haber hallado buena concordancia entre experimentos y la predicción.

\section{FORMULACIÓN ENTÁLPICA}

Debe existir una relación uno a uno entre entalpia y temperatura para que una formulación de este tipo sea significativa, la entalpia se vuelve la variable dependiente primaria y la temperatura la dependiente secundaria y esto permite también que la ecuación gobernante aplique a ambas fases.

\section{EL MÉTODO}

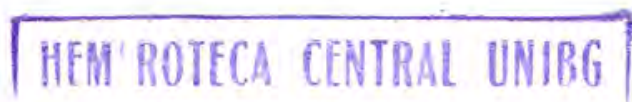

depende del sistema de coordenadas. Las condiciones de frontera de las superficies expuestas comúnmente halladas en congelación y descongelación de alimentos son las de temperatura fija y tipos convectivos. Los casos unidimensionales simétricos, se usa una condición de frontera adicional que es flujo de calor cero en el centro de simetria. La condición inicial común es una distribución de temperatura conocida a través de todo el alimento.

Ecuación gobernante:

$$
\frac{\partial H}{\partial t}=\frac{1}{r^{m}} \cdot \frac{\partial}{\partial r}\left[r^{m} \cdot K \frac{\partial T}{\partial r}\right]
$$

donde $m=0,1$ y 2 para coordenadas rectangular, cilindrica y esférica

\section{Condiciones de Frontera}

- en la superficie

$$
\begin{array}{ll}
K . \frac{\partial T}{\partial r}=h\left(T_{a}-T_{s}\right) & \text { convectiva } \\
T=T_{5} & \text { temperatura fija }
\end{array}
$$

donde $\mathrm{a}=$ ambiente; $\mathrm{s}=$ superficie

- en el centro

$$
\text { K. } \frac{\partial T}{\partial r}=0
$$

- condición inicial

$$
T-T,
$$

$l=$ inicial

Las relaciones siguientes expresan la dependencia de la temperatura y de la conductividad térmica respecto de la entalpia

$$
\begin{aligned}
& T=T(H) \\
& K=K(H)
\end{aligned}
$$

Consiste de una ecuación gobernante, condiciones iniciales y de frontera y las propiedades de los alimentos. La primera se obtiene del balance de calor sobre un pequeño elemento del alimento y su forma y pueden estar en forma de expresiones analiticas o como tablas de interpolación, prefiriéndose generalmente las segundas por simplicidad. 


\section{EL ESQUEMA DE DIFERENCIAS FINITAS}

El balance de calor para los nodos de una red de elementos finitos origina las siguientes ecuaciones que constituyen el esquema de Mannaperuma: para un elemento de sección transversal de área unitaria en un nodo interior $\mathrm{i}$ entre niveles de tiempo $\mathrm{j}$ y $\mathrm{j}+1 \mathrm{y}$ considerando factores de volumen y área se tiene

$$
\begin{gathered}
H_{i}^{\prime}{ }^{\prime}=H_{i}^{\prime}+\frac{\Delta t}{V_{1}[\Delta r]^{2}}\left(a _ { i + \frac { 1 } { 2 } } K _ { i + \frac { 1 } { 2 } } \left[T_{i+1}^{j}-\right.\right. \\
\left.\left.T_{i}^{\prime}\right]-a_{i-\frac{1}{2}} K_{i-\frac{1}{2}}\left[T_{i}^{\prime}-T_{i-1}^{\prime}\right]\right)
\end{gathered}
$$

para un elemento en el nodo o

$$
H_{0}^{j+1}=H_{0}^{j}+\frac{\Delta t}{V_{0}[\Delta r]^{2}}\left[a_{\frac{1}{2}} K_{\frac{1}{2}}\left(T_{l}^{\prime}-T_{0}^{j}\right)\right]
$$

para condición de frontera convectiva con temperatura de superficie especificada el balance sobre el elemento en el nodo $n$ es

$$
\begin{aligned}
H_{n}^{j+1} & =H_{n}^{j}+\frac{\Delta}{V_{n}[\Delta r]^{2}}\left[a_{n} h \Delta r\left(T_{a}^{j}-T_{n}^{j}\right)\right. \\
& \left.-a_{n-\frac{1}{2}} K_{n-\frac{1}{2}}\left(T_{n}^{j}-T_{n-1}^{j}\right)\right]
\end{aligned}
$$

Las entalpías nodales se actualizan con las tres ecuaciones anteriores y luego se calculan la temperatura y la conductividad térmica en los nodos mediante las relaciones (5) y (6). La conductividad térmica se calcula a la mitad de los nodos por interpolación lineal de los valores de los nodos situados a ambos lados. Así se completa el procedimiento de actualización que luego puede repetirse. Los factores de volumen y área difieren en los 3 sistemas de coordenadas. La Tabla 1 de Mannaperuma contiene expresiones adecuadas para estas expresiones.

Tabla 1. Factores de volúmen y área según sistemas de coordenadas.

\begin{tabular}{|llll|}
\hline & Rectangular & \multicolumn{1}{c|}{ Cilíndricas } & \multicolumn{1}{c|}{ Esféricas } \\
\hline$V_{0}$ & $1 / 2$ & $1 / 8$ & $1 / 24$ \\
$V_{i}$ & 1 & $i$ & $i^{2} \pm 1 / 12$ \\
$V_{n}$ & $1 / 2$ & $1 / 2(n-1 / 4)$ & $1 / 2\left(n^{2}-n / 2+1 / 12\right)$ \\
$a_{1 / 2}$ & 1 & $1 / 2$ & $1 / 4$ \\
$a_{i t 1 / 2}$ & 1 & $i \pm 1 / 2$ & $(i \pm 1 / 2)^{2}$ \\
$a_{n}$ & 1 & $n$ & $n^{2}$ \\
\hline
\end{tabular}

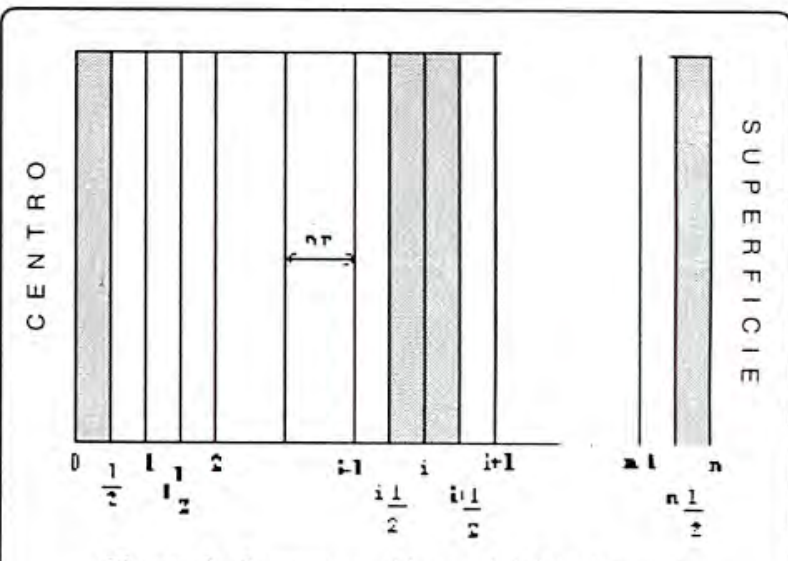

Figura 1. Representación nodal del alimento.

\section{ESTABILIDAD}

El criterio es similar que para la formulación del calor específico aparente, pero debe satisfacerse en cada nodo para cada incremento de tiempo para asegurar estabilidad global debido a la dependencia de las propiedades térmicas de la temperatura. Los dos factores de área y dos conductividades en la ecuación (7) complican la expresión de estabilidad para los nodos interiores. Mannaperuma soluciona esto promediando los dos pares de valores y obtiene la siguiente ecuación:

$$
\begin{gathered}
H_{i}^{\prime+1}=H_{i}^{j}+\frac{a_{i} K_{i} \Delta t}{V_{i}[\Delta r]^{2}}\left[\left(T_{i-1}^{j}-T_{i}^{j}\right)\right. \\
\left.=\quad\left(T_{i}^{j}-T_{i+1}^{j}\right)\right]
\end{gathered}
$$

donde:

$$
\begin{aligned}
& a_{1}=\frac{1}{2}\left[a_{i-\frac{1}{2}}+a_{i+\frac{1}{2}}\right] \\
& K_{i}=\frac{1}{2}\left[K_{i-\frac{1}{2}}+K_{i+\frac{1}{2}}\right]
\end{aligned}
$$

Los términos de la entalpía en la derivación del criterio de estabilidad, están expresados en función del calor específico aparente y reacomodados así:

$$
\frac{C_{i}^{j+1}}{C_{i}^{j}} T_{i}^{j+1}=S T_{i+1}^{j}+(1-2 S) T_{i}^{j}+S T_{i-1}^{j}
$$


donde:

$$
S=\frac{a_{i} K_{i} \Delta t}{V_{i} C_{i}^{j}[\Delta r]^{2}}
$$

La estabilidad se mantiene si el coeficiente del término del medio del lado derecho es siempre positivo. El criterio de estabilidad de la ecuación (11) puede escribirse asi:

$$
S \leq \frac{1}{2}
$$

que puede reescribirse:

$$
\frac{\Delta t}{[\Delta r]^{2}} \leq \frac{\sigma_{i}}{\alpha_{i}}
$$

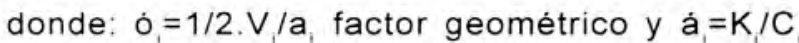
difusividad térmica aparente.

La desigualdad (13) expresa el límite superior del parámetro de estabilidad en el lado derecho como la relación entre dos factores uno de los cuales depende sólo de la geometria en tanto que el otro es una propiedad del alimento. Los criterios de estabilidad para los nodos central y en la superficie se derivan de la misma manera aunque no requieren se promedien los factores geométricos ni las conductividades térmicas. Asi:

nodo central:

$$
\sigma_{0}=\mathrm{V}_{0} / \mathrm{a}_{1 / 2} ; \alpha_{0}=\mathrm{K}_{1 / 2} / \mathrm{C}_{0}
$$

nodo superficial:

$$
\sigma_{n}=\left[V n /\left(B a_{n}+a_{n-1 / 2}\right)\right] \propto n=\left(K_{n-1 / 2}\right) / C_{n}
$$

donde : $B=h \Delta r / K_{n-1 / 2}$

En la Tabla 2 aparecen resumidos los factores geométricos en los tres sistemas coordenados presentados por mannaperuma. Puede verse que el factor geométrico toma valores minimos en el nodo superficial y en el central. La difusividad térmica aparente toma valor máximo a las más bajas o más altas temperaturas lo que ocurre otra vez en el nodo superficial y central en congelación y descongelación.
En consecuencia, es generalmente adecuado satisfacer el criterio de estabilidad en los nodos superficial y central para asegurar estabilidad en todos los nodos.

\section{EL PROGRAMA COMPUTACIONAL}

Está escrito en Basic como parte de la tesis doctoral de Mannaperuma, puede manejar formulaciones unidimensionales para congelación y descongelación en los tres sistemas coordenados.

Debe ingresarse como datos entalpia volumétrica y conductividad del alimento sobre el rango de temperatura aplicable. Otros datos a ingresar son: tipo de proceso, congelación, descongelación; geometria; dimensión principal; condición de frontera, temperatura media y coeficiente de transferencia de calor; temperatura inicial; temperatura final en el centro e intervalo de tiempo para visualización en pantalla.

Otros datos a ingresar son: tipo de proceso, congelación, descongelación; geometria; dimensión principal; condición de frontera, temperatura media y coeficiente de transferencia de calor; temperatura inicial; temperatura final en el centro e intervalo de tiempo para visualización en pantalla.

En intervalos regulares, se calcula el incremento de tiempo más grande posible que satisfaga el criterio de estabilidad, proceso que ayuda a reducir el tiempo de computación a la vez que asegura estabilidad.

\section{TABLAS DE ENTALPIA Y PROPIEDADES FISICAS $Y$ TABLAS MATRICIALES}

Se prepararon luego las tablas de interpolación de entalpias y las tablas de propiedades termofisicas para abalón, caracol, bacalao y carne de vacuno utilizando un subprograma del sistema Mannaperuma. Todas ellas útiles para la simulación posterior acomodada en tablas matriciales en las que las temperaturas por cada intervalo de tiempo escogido, aparecen en filas y bajo columnas que corresponden a la posición nodal.

hehrofecea central unibg 
Tabla 2. Factor geométrico ó en los tres sistemas coordenados.

\begin{tabular}{|lccc|}
\hline & Rectangular & Cilindrico & Esférico \\
\hline nodo 0 & $1 / 2$ & $1 / 4$ & $1 / 6$ \\
nodo $\mathrm{i}$ & $1 / 2$ & $1 / 2$ & $1 / 2\left[1+1 / 12^{2}\right]$ \\
nodo $n$ & $1 /[2(1+B)]$ & $\frac{1[n-1 / 4]}{2 n(1+B)-1}$ & $\frac{1\left[n^{2}-n / 2+1 / 12\right]}{2 n^{2}(1+B)-2 n+1 / 2}$ \\
\hline
\end{tabular}

\section{CURVAS DE CONGELACIÓN Y DESCONGELACIÓN SIMULADAS VERSUS CURVAS EXPERIMENTALES CORRESPONDIENTES}

De las tablas matriciales obtenidas en largas sábanas de papel se tomaron los datos de congelación y descongelación para poder compararlos con sus correspondientes experimentales y estar en condiciones de verficar la bondad de aproximación del método cuando aplicado a productos reales.

\section{CÁLCULO DE LOS TIEMPOS DE CONGELACIÓN Y DESCONGELACIÓN}

Fue un proceso fácil de realizar luego que se hubo preprogramado que la simulación se detuviera en cada caso cuando la temperatura en el centro del producto correspondiente alcanzara $-18^{\circ} \mathrm{C}$. Sólo había que leer directamente el tiempo transcurrido de la tabla matricial. Esto por supuesto, permitió también comparar con tiempos experimentalmente medidos por algunos investigadores de renombre.

\section{LA ESTIMACIÓN DEL ERROR}

Lo usual en este tipo de trabajos es hacer una comparación cuantitativa entre los tiempos calculados con la simulación y los experimentales en base a la definición del porcentaje de error de predicción como sigue:

t. simulado - t. experimental $\times 100$ tiempo experimental

\section{RESULTADOS Y DISCUSIÓN}

\section{SIMULACIÓN DE PROPIEDADES}

En las páginas finales aparece una de las tablas de interpolación de propiedades físicas que son el resultado de la ejecución del programa PROP4.EXE; para cada especie o producto debe ingresarse algunos datos dependiendo de la opción escogida para la simulación de propiedades. La opción 1 requiere la entrada de arreglos de propiedades como entalpía volumétrica y conductividades térmicas a diferentes temperaturas; la opción 2 necesita el ingreso de propiedades del alimento no congelado y la opción 3 , su composición química. En el presente estudio, se utilizaron las opciones 2 y 3.

La selección de ambas lleva a subopciones que permiten el ingreso de datos vía teclado y también el uso de la base de datos acompañante; la porosidad del alimento es por ejemplo uno de los valores que se puede decidir ingresar por teclado, asumir como cero o calcularse en base a la densidad. Para el agua no congelable se cuenta con rangos proporcionados por el programa que la relacionan con el contenido de sólidos y basta corregirlos con los factores adecuados para estimar el porcentaje de agua no congelable.

Otrodelospasosnecesarioseslaentradadel punto inicial de congelación y señalar que el objetivo es preparar un archivo en disco que puede ser usado luegoenlasimulacióndecongelación/descongelación.

Debe indicarse asímismo el rango aproximado de operacióndelprocesodecongelación/descongelación. Las dos primeras tablas de cada especie o producto enlosanexos sonlasversionesimpresas delosarchivos ASCII *LIST y *PROP respectivamente (por ejemplo, CARALIST Y CARAPROP para caracol; BACALIST, BACAPROP para bacalao, etc).

Los datos ingresados son los que se aprecian en la Tabla 3.

\section{SIMULACIÓN DE CONGELACIÓN/DESCONGELACIÓN}

\section{Tablas Matriciales}

Se usó exclusivamente el programa EN1D4.exe para situaciones unidimensionales (hay otros dos programas que cubren las bi y trimensionales); el resultado son las terceras tablas que para cada producto aparecen en los anexos. Los datos ingresados aparecen también en la Tabla 3.

\section{Curvas y Tiempos de Congelación}

Enlas gráficas másadelanteseaprecianlascurvas experimentales (tomadas de la bibliografía especializada) y las curvas simuladas con el programa de 
Mannaperuma; se considera el puré de papas por tratarse de una sustancia aceptada como homogénea y de prueba en este tipo de estudios; los demás productos por estimarse que constituyen buenos experimentos.

En el caso de puré de papas, se aprecia una demora en la zona de enfriamiento, retraso en el inicio de la congelación y también en la zona de temperado habiendo una discrepancia del $8,1 \%$ entre el tiempo de congelación experimental (74 $\mathrm{min})$ y el de simulación (80 min ).

En el caso de abalón, se elaboró sólo la curva de simulación, pues no se encontró una experimental para hacer la comparación, contándose únicamente con un tiempo de $57 \mathrm{~min}$. calculado teóricamente con la fórmula de Planck contra los 82 minutos de la simulación constituyendo de todas maneras una referencia aunque se sabe ya que Planck tiende a subestimar los tiempos de congelación.

Para caracol, la conducta de la curva simulada sigue la misma tendencia que en el caso de puré, sobrestimación inicial, retraso en el inicio de la congelación y prolongación en la zona de temperado aunque el tiempo de congelación obtenido es aproximadamente el mismo (30 min).

En el caso de bacalao, hay coincidencia casi perfecta al inicio del enfriamiento y al inicio de la congelación, luego una ligera aceleración en la zona de máxima remoción de calor latente,luego una prolongación bastante extensa en la zona de temperado arrojando una considerable discrepancia $(30,9 \%)$ en los tiempos de congelación, 84 minutos del experimento y 110 para la simulación.

Para carne de res magra ocurre lo mismo que para puré, caracol y bacalao, demora en el enfriamiento $e$ inicio de la congelación y gran prolongación en la zona de temperado arrojando una discrepancia notoria en los tiempos de congelación, 32 minutos experimental y 48 minutos simulación ( $50 \%$ error).

Respecto a la descongelación, sólo se usó como comparación carne de res magra observándose poca unformidad en la forma de las curvas; la simulación muestra incremento más gradual hasta los $-5 C$ y más abrupta a partir de esa temperatura arrojando tiempos de descongelación de 44 min experimental y $36 \mathrm{~min} \mathrm{la}$ simulación (-18\%).
Tabla 3. Datos Utilizados en la simulación de propiedades y en la simulación de Congelación/Descongelación.

\begin{tabular}{|c|c|c|c|c|c|c|c|}
\hline \multicolumn{8}{|c|}{ Simulación de Propiedades } \\
\hline \multirow{2}{*}{$\begin{array}{l}\text { Opción } 2 \\
\text { Producto }\end{array}$} & \multirow{2}{*}{$\begin{array}{l}\tilde{n}(\mathrm{nc}) \\
{[\mathrm{kg} / \mathrm{m} 3]}\end{array}$} & $\mathrm{Cp}(\mathrm{nc})$ & $\mathrm{K}(\mathrm{nc})$ & \multirow{2}{*}{$\begin{array}{l}\text { Tinic } \\
{[C]}\end{array}$} & \multirow{2}{*}{$\begin{array}{l}\mathrm{H} 2 \mathrm{O} \\
\text { (dec.) }\end{array}$} & \multirow{2}{*}{$\begin{array}{l}\mathrm{H} 2 \mathrm{O} \mathrm{nc} \\
\text { (dec.) }\end{array}$} & \multirow{2}{*}{$\begin{array}{cc}\text { TI } & \text { c.t. } \\
{[\mathrm{C}]} & {[\mathrm{C}]}\end{array}$} \\
\hline & & $\mathrm{I} / \mathrm{Kg} \cdot \mathrm{K}]$ & {$[\mathrm{W} / \mathrm{m}$. } & & & & \\
\hline puré papas & & 3634 & 0,5 & 7.5 & 0.82 & 0.0387 & $-1,7 \cdot-18 \mathrm{c}$ \\
\hline Opción 3 & \multicolumn{2}{|l|}{ Abalón } & \multicolumn{2}{|c|}{ Caracol } & Bacalao & \multicolumn{2}{|c|}{ Carne res magra } \\
\hline $\mathrm{H}_{2} \mathrm{O}$ & \multicolumn{2}{|l|}{0.758} & \multicolumn{2}{|c|}{0.70} & 0,80 & \multicolumn{2}{|c|}{0,77} \\
\hline Proteína & \multicolumn{2}{|l|}{0,187} & \multicolumn{2}{|c|}{0.18} & 0,15 & \multicolumn{2}{|c|}{0.22} \\
\hline Grasa & \multicolumn{2}{|l|}{0,005} & \multicolumn{2}{|c|}{0,05} & 0,03 & \multicolumn{2}{|c|}{0.00} \\
\hline Carbohidratos & \multicolumn{2}{|l|}{0,034} & \multicolumn{2}{|l|}{0,00} & 0.00 & \multicolumn{2}{|c|}{0.00} \\
\hline Fibra & \multicolumn{2}{|l|}{0,000} & \multicolumn{2}{|c|}{0,00} & 0,00 & \multicolumn{2}{|c|}{0,00} \\
\hline Ceniza & \multicolumn{2}{|l|}{0,000} & \multicolumn{2}{|c|}{0,07} & 0.02 & \multicolumn{2}{|c|}{0.01} \\
\hline Porosidad & \multicolumn{2}{|l|}{0.000} & 0.00 & & 0,00 & 0,00 & \\
\hline $\mathrm{H}_{2} \mathrm{O} \mathrm{nc}$ & $0,24 \mathrm{cs}$ & & $0,24 \mathrm{c}$ & & $0.24 \mathrm{cs}$ & & $45 \mathrm{cs}$ \\
\hline $\mathrm{T}_{1}[\mathrm{C}]$ & $-1,7$ & & $-2,0$ & & $-2,2$ & -5 & \\
\hline Rango Temp. $[\mathrm{C}]$ & $10 a \cdot 18$ & & $18 \mathrm{a}$ & -15 & 16 a. 18 & & $a-15$ \\
\hline Simulación de $C$ & Congelacic & ión / D & Descon & ngelación & & & \\
\hline & Puré & & alón & Caracol & Bacalao & Carne re: & s magra \\
\hline & & & ingelaci & & & Cong. & Desc. \\
\hline Configurac. & placa i. & pla & ica i. & esfera & placa i. & placa i & \\
\hline espesor tot. & $50 \mathrm{~mm}$ & & $6 \mathrm{~mm}$ & $42 \mathrm{~mm}$ & $50.8 \mathrm{~mm}$ & $25,4 \mathrm{~mm}$ & \\
\hline Tinic $[\mathrm{C}]$ & 7.5 & 10 & & 18 & 15,5 & 10 & -20 \\
\hline c.t. & conv. & con & & conv. & conv. & conv. & conv. \\
\hline $\mathrm{h}[\mathrm{W} / \mathrm{m} 2 \mathrm{~K}]$ & 250 & 22 & & 75 & 102,3 & 80 & 5 \\
\hline Tamb & -24 & .35 & & -27 & -27.5 & -30 & 22 \\
\hline c.t. & $-18 c$ & -18 & & $-15 c$ & $.18 c$ & $-18 c$ & 10Teq \\
\hline i.t. [s] & 120 & 12 & & 60 & 120 & 60 & 120 \\
\hline
\end{tabular}

nc $=$ no congelado/no congelable

$\mathrm{T}_{1}=$ temperatura inicial de congelación

c.t. = criterio de terminación de simulación

c $=$ temp. en el centro

Teq = temperatura equivalente

cs $\quad=$ contenido de sólidos

c.f. = condición de frontera

$\mathrm{h}=$ coef. transf. calor

Tamb $=$ temperatura del ambiente (medio congelante)

i.t. $=$ intervalo de tiempo para salida de resultados

La Tabla 4 muestra el resúmen de los tiempos experimentales y simulados. 
Tabla 4. Resúmen de Tiempos de Congelación/Descongelación [min].

\begin{tabular}{|l|c|c|c|}
\hline \multicolumn{1}{|c|}{ Producto } & $\begin{array}{c}\text { t.exp. } \\
{[\mathrm{min}]}\end{array}$ & $\begin{array}{c}\text { t.sim } \\
{[\mathrm{min}]}\end{array}$ & $\begin{array}{c}\text { error } \\
{[\%]}\end{array}$ \\
\hline Congelación & 74 & 80 & 8,1 \\
puré papas & $\ddots$ & 82 & $-\because$ \\
Abalón & 30 & 30 & 0,0 \\
Caracol & 84 & 110 & 30,9 \\
Bacalao \\
carne r.m. & 32 & 48 & 50,0 \\
\hline Descongelación & & & \\
carne r.m. & 44 & 36 & $-18,0$ \\
\hline
\end{tabular}

Debe aclararse que se han comparado experimentos puntuales que en su mayoría no detallan las condiciones utilizadas especialmente respecto a los valores de las propiedades térmicas y el coeficiente de transferencia de calor, indican cómo obtuvieron esos valores pero no los presentan, salvo el caso de bacalao en filetes en el que se logra una similitud muy apreciable en la curva de congelación hasta que se llega a la zona de temperado en que la prolongación del tiempo de congelación se hace notoria. Los experimentos son pocosyno hay razón suficientepara sostener que el método de Mannaperuma no es suficientemente exacto. Es necesario comparar con muchos más experimentos que ofrezcan suficiente detalle sobre su realización.

En los esquemas de diferencias finitas tipo Lees, modificación de Crank-Nicholson, totalmente implícitos, totalmente explícitos y explícitos con transformación de entalpía, las diferencias tienen lugar debido a dos causas, el error de truncación que es menor en el primero de los mencionados por la mejor aproximación de la derivada del tiempo. También es importante el nivel de tiempo en el cual se evalúan las propiedades térmicas. Idealmente, la derivada del tiempo se mantiene constante en el nivel de tiempo del punto medio (i) y las propiedades se evalúan en el nivel de punto medio; en el resto de la ecuación de conducción, las derivadas respecto al espacio y las propiedades deberían evaluarse en el nivel de tiempo de punto medio en el esquema de diferencias; esto puede originar error sistemático. Esto puede reducirse a medida que se reduce el paso del incremento de tiempo.
Otra posible explicación de la sobrestimación del tiempo de congelación puede estar en el hecho que la asunción del programa de Mannaperuma se basa en que las funciones de las propiedades térmicas son dependientes de la temperatura pero no de la posición lo que puede causar predicciones erróneas de temperatura especialmente en materiales no homogéneos.

Fig. 1. Congelación de Puré de Papas. Placa de $5 \mathrm{~cm}$. espesor.

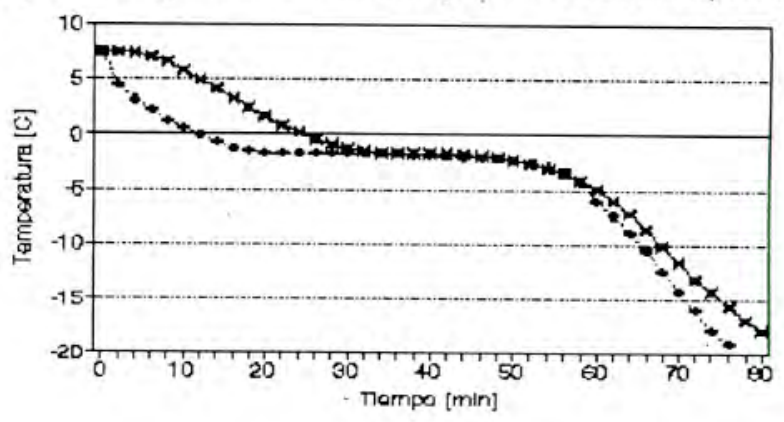

- Experimental \#- Birrulacion

Fig. 2. Congelación de Abalón en aire forzado.

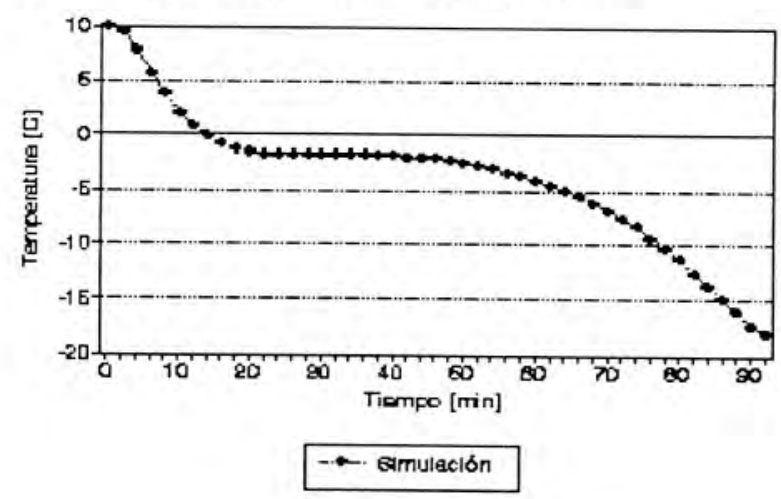

Fig. 3. Congelación de Caracol (Thais chocolata).

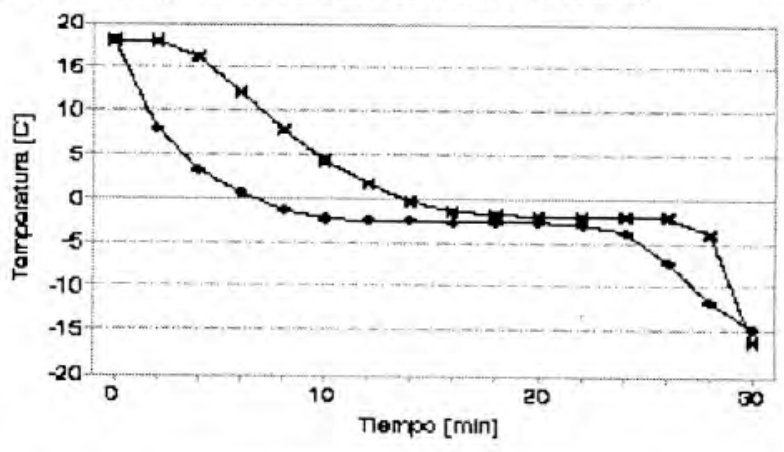

Experimental $\rightarrow$ - Gimubaibn 
Fig. 4. Congelación de bacalao. Filetes 5,08 cm espesor

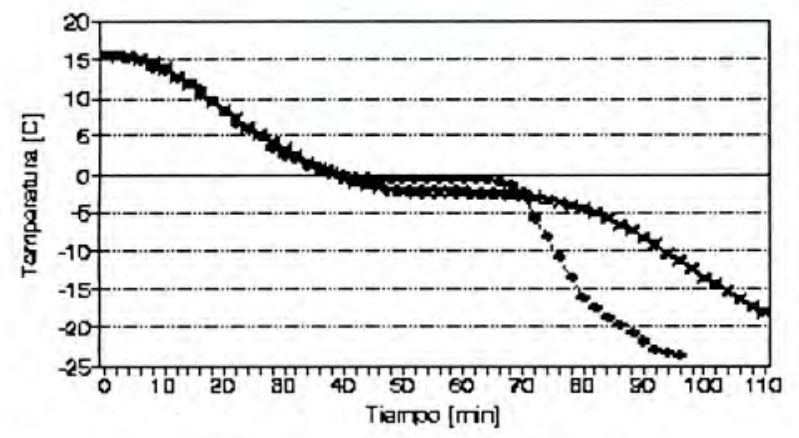

Fig. 5. Congelación de Carne de res en filetes de $I^{2}$.

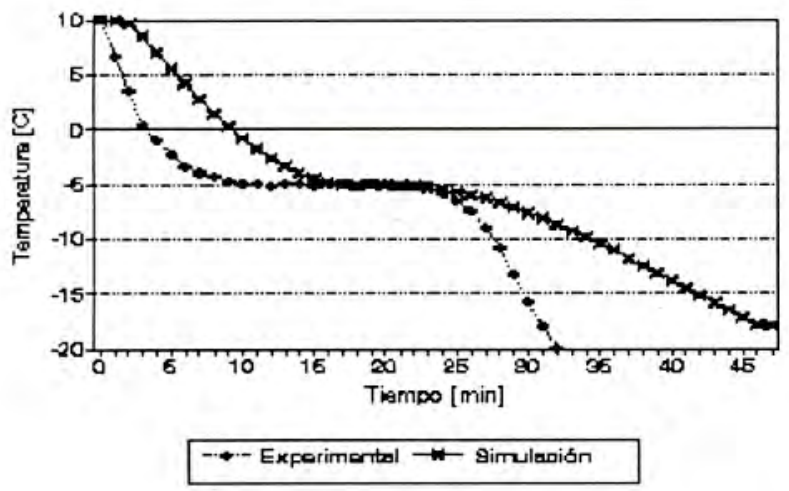

Fig. 6. Descongelación de carne de res magra magra, filetes $2,5 \mathrm{~cm}$.

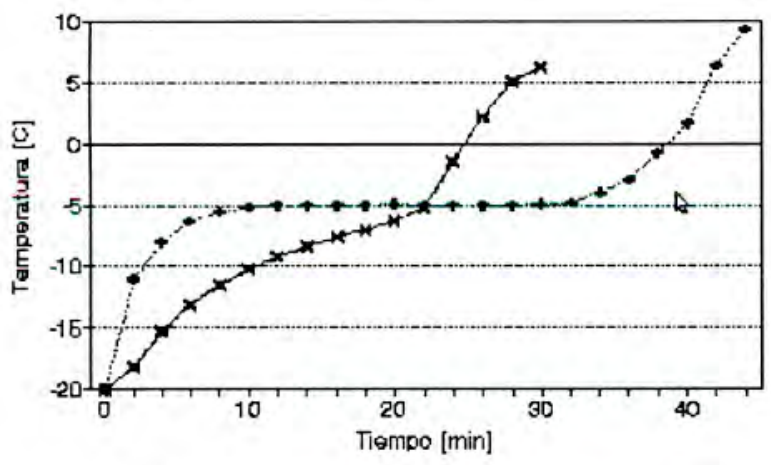

- +.- Experimental $\rightarrow$ Simulacion

\section{CONCLUSIONES}

- ElPrograma de Mannaperuma es de fácil uso pero necesita contarse con información suficientemente sólida y claramente disponible para aplicaciones a datos experimentales.

- Es sencillo elaborar tablas de propiedades simuladas y utilizarlas en la simulación de procesos de congelación / descongelación.

- Elprograma de Mannaperuma tiende a sobrestimar aparentemente los tiempos de congelación para criterios de terminación de temperatura en el centro y subestimar en descongelación para criterios de terminación de proceso basados en temperatura de equilibrio en paquetes alimenticios reales (no homogéneos).

- La exactitud del método no ha sido probada suficientemente por lo que debe seguirsele considerando como una posibilidad para las predicciones sobre todo por evitar el "salto" de la capacidad calórica aparente.

\section{RECOMENDACIONES}

- Reunir mayor número de datos experimentales utilizables para probar la exactitud del método Mannaperuma.

- Buscar un método numérico estandar con el cual hacer una comparación de Mannaperuma y cualquier otro método de interés.

- Específicamente ensayar Mannaperuma con diferentes temperaturas iniciales, espesores de placas o diámetros de cilindros o esferas así como con valores del coeficiente de transferencia de calor confiables.

- Combinar lo anterior con reducciones en el paso del incremento de tiempoy reajustar esos incrementos cuando sea necesario. 


\section{REFERENCIAS BIBLIOGRÁFICAS}

CLELAND A., EARLE R., CLELAND D., The effect of Freezing rate on the accuracy of numerical freezing time prediction. Int. J. Refrig. 5:294, 1982

CLELAND A., EARLE R., Assessment of Freezing Time Prediction Methods. J. Food Science, Volume 49 (1984)

HELDMAND., Computer Simulation of Food Freezing Processes, Proc IV Int. Congress Food Sci. and Technol.Vol IV,p. $397-$ 406 (1974)

HELDMAN D., Factors Influencing Food Freezing Rates, Food Technology (1983), 37(4), 103-109.

MANNAPERUMA, J., SINGH P., Prediction of Freezing and Thawing Times of Foods Using a Numerical Method Based on Enthalpy Formulation. J Food Science volume 53, No 21988

Thermal Properties of the Food Material (Caracol) tabulated at selected temperatures.

\begin{tabular}{|c|c|c|c|c|}
\hline $\begin{array}{c}\text { Temperature } \\
\text { deg C }\end{array}$ & $\begin{array}{c}\text { Density } \\
\mathbf{k g} / \mathrm{m} 3\end{array}$ & $\begin{array}{c}\text { Sp. heat } \\
\text { J/kg-K }\end{array}$ & $\begin{array}{c}\text { Enthalpy } \\
\mathrm{J} / \mathbf{k g}\end{array}$ & $\begin{array}{c}\text { Th.Conduc- } \\
\text { tivity } \\
\mathbf{W} / \mathbf{m}-\mathbf{K}\end{array}$ \\
\hline-17.70 & 1032 & 3389 & 57790 & 1.634 \\
\hline-14.50 & 1034 & 4064 & 69644 & 1.579 \\
\hline-11.30 & 1036 & 5377 & 84531 & 1.508 \\
\hline-9.70 & 1038 & 6565 & 194034 & 1.461 \\
\hline-8.10 & 1040 & 8521 & 105984 & 1.402 \\
\hline-6.50 & 1044 & 12086 & 122181 & 1.320 \\
\hline-5.70 & 1047 & 15092 & 132985 & 1.265 \\
\hline-4.90 & 1050 & 19686 & 146770 & 1.193 \\
\hline-4.10 & 1055 & 27221 & 165277 & 1.095 \\
\hline-3.70 & 1059 & 32948 & 177258 & 1.031 \\
\hline-3.30 & 1063 & 40880 & 191940 & 0.952 \\
\hline-2.90 & 1068 & 52315 & 210437 & 0.850 \\
\hline-2.70 & 1071 & 60029 & 221646 & 0.788 \\
\hline-2.50 & 1075 & 69668 & 234583 & 0.716 \\
\hline-2.30 & 1080 & 81930 & 249696 & 0.632 \\
\hline-2.20 & 1083 & 89349 & 258251 & 0.583 \\
\hline-2.10 & 1086 & 97856 & 267604 & 0.530 \\
\hline-2.00 & 1089 & 107671 & 277870 & 0.471 \\
\hline 0.00 & 1089 & 3463 & 284797 & 0.474 \\
\hline 9.00 & 1089 & 3467 & 316006 & 0.485 \\
\hline-18.00 & 1090 & 3471 & 347281 & 0.497 \\
\hline
\end{tabular}

SANDERS H., A Computer Programme for the Numerical Calculation of Heating and Cooling Processes in Blocks of Fish, Advances in Fish Science and Technology Torry, Aberdeen 1979.

SUCCAR J., HAYAKAWA K., A Response Surface Method for the estimation of convective and radiative heat transfer coefficients during freezing and thawing of foods, J. Food Sci. $51: 1314$ (1986)

WILSONH., SINGH P., Numerical Simulation of Individual Quick Freezing of Spherical Foods, Int. J. Refrig. 10:149, 1987

YEN-CON HUNG, Prediction of Cooling and Freezing Times, paper presented during the Refrigerated and Frozen Food Division Program "Technical Advances in Chilled and Frozen Foods", at the 1989 Annual Meeting of the Institute of Food Technologists, Chicago, III, June 25-29 1989.

Thermal Properties of the Food Material (Caracol) tabulated at equal increments of volumetric enthalpy.

\begin{tabular}{|c|c|c|}
\hline $\begin{array}{c}\text { Temperature } \\
\text { Deg C }\end{array}$ & $\begin{array}{l}\text { Enthalpy } \\
\text { MJ/m3 }\end{array}$ & $\begin{array}{c}\text { Thermal } \\
\text { Conductivity } \\
\text { W/m-K }\end{array}$ \\
\hline-17.70 & . 59.7 & 1.634 \\
\hline-13.71 & 75.9 & 1.561 \\
\hline-10.59 & 92.0 & 1.487 \\
\hline-8.36 & 108.2 & 1.412 \\
\hline-6.79 & 124.4 & 1.335 \\
\hline-5.62 & 140.6 & 1.258 \\
\hline-4.79 & 156.8 & 1.180 \\
\hline-4.16 & 173.0 & 1.102 \\
\hline-3.66 & 189.2 & 1.023 \\
\hline-3.27 & 205.4 & 0.945 \\
\hline-2.96 & 221.6 & 0.866 \\
\hline-2.70 & 237.8 & 0.787 \\
\hline-2.48 & 253.9 & 0.708 \\
\hline-2.30 & 270.1 & 0.629 \\
\hline-2.14 & 286.3 & 0.550 \\
\hline-2.00 & 302.5 & 0.471 \\
\hline 2.26 & 318.7 & 0.477 \\
\hline 6.54 & 334.9 & 0.482 \\
\hline 10.81 & 351.1 & 0.488 \\
\hline 15.08 & 367.3 & 0.493 \\
\hline
\end{tabular}


Simulation of Freezing /Thawing of 1-D Objects (Caracol).

Press $[\mathrm{F} 10]$ to terminate

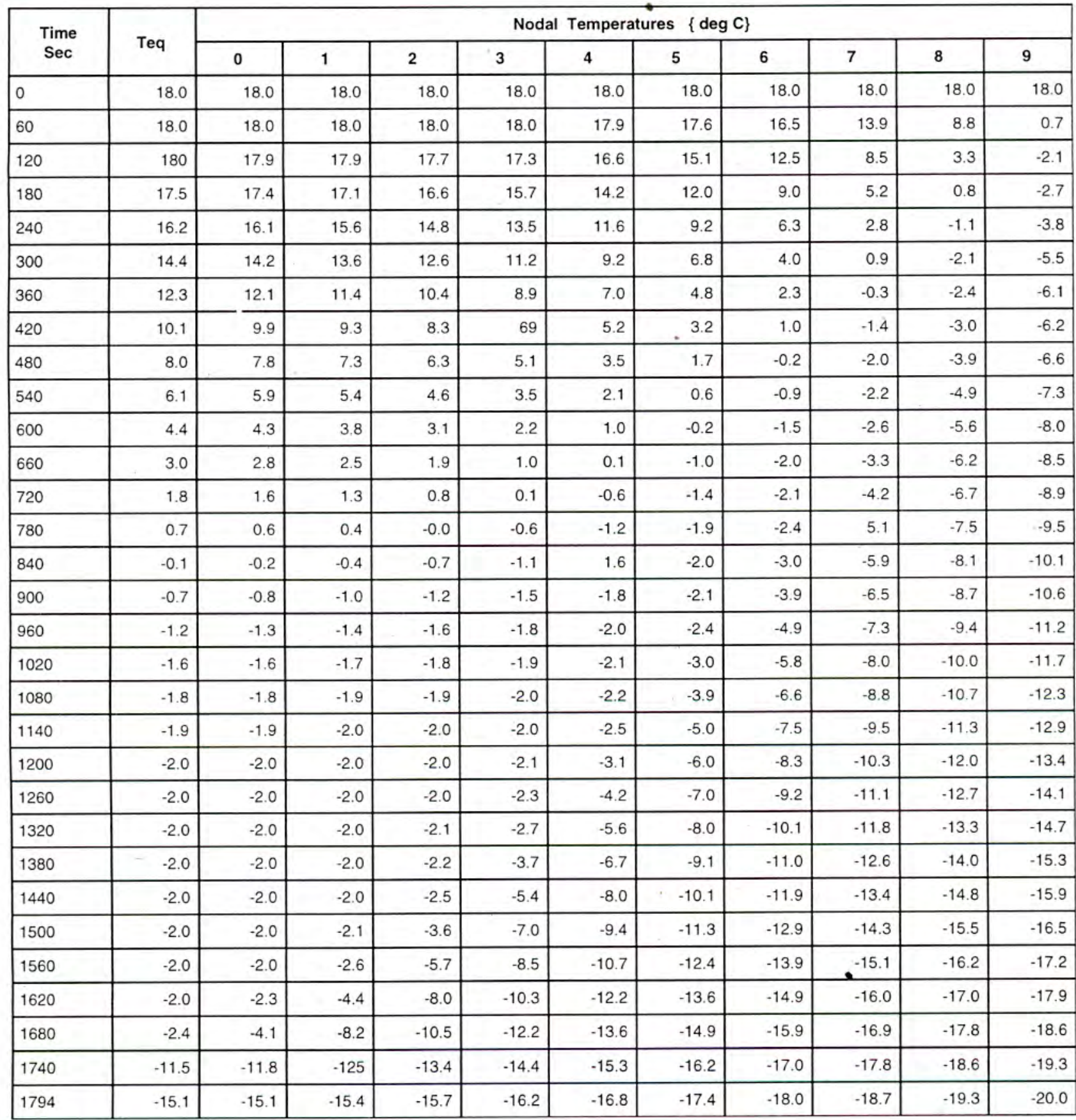

Process duration

Execution time

Energy removal

Enthalpy decrease

Energy balance error
End of simulation

1793.751 Sec

00:00:35 hh:mm:ss

$12381.56 \mathrm{~J}$

$12468.2 \mathrm{~J}$

-.6949317 Percent 